Xenia Augustin*, Michael Kircher, Birgit Stender, Thomas Bluth, Marcelo Gama de Abreu, and Olaf Dössel

\title{
Estimating regional pulmonary blood flow in EIT with regularized deconvolution with a Tikhonov regularization
}

\author{
Comparing the estimation of pulmonary blood flow in EIT with regularized deconvolution to \\ the maximum slope method
}

https://doi.org/10.1515/cdbme-2020-3016

\begin{abstract}
Electrical Impedance Tomography (EIT) is a clinically used tool for bed-side monitoring of ventilation. Previous work also showed a high potential for lung perfusion monitoring with indicator-enhanced EIT. However, many research questions have yet to be answered before it can be broadly applied in clinical everyday life. The goal of this work is to evaluate a new method to improve EIT perfusion measurements. Pulmonary hemodynamic transfer functions were estimated using regularized deconvolution with Tikhonov regularization to estimate spatial perfusion parameters. The final comparison between EIT images and PET scans showed a median correlation of 0.897 for the images which were reconstructed using the regularized deconvolution. In comparison the previously used maximum slope method led to a median correlation of 0.868 .
\end{abstract}

Keywords: EIT, perfusion measurement, rBF, Tikhonov regularization, maximum slope, image reconstruction.

\section{Introduction}

One of the most common complications in critical ill patients is Acute Respiratory Distress Syndrome (ARDS) [1]. ARDS is a pulmonary disease which can lead to insufficient blood oxygenation and reduced expansibility of the lungs. In the long term it can also lead to the collapse of the lungs. The mortality

*Corresponding author: Xenia Augustin, Institute of Biomedical Engineering, KIT, Fritz-Haber-Weg 1, Karlsruhe, Germany, e-mail: publications@ibt.kit.edu

Michael Kircher, Olaf Dössel, Institute of Biomedical

Engineering, KIT, Karlsruhe, Germany

Birgit Stender, Drägerwerk AG \& Co. KGaA, Lübeck

Thomas Bluth, Marcelo Gama de Abreu, Pulmonary Engineering Group Dresden, Dep. of Anesthesiology and Intensive Care Medicine, University Hospital \& Technische Universität, Dresden, Germany rate is $27-45$ percent [2]. ARDS can occur as a result of direct lung damage or as a systemic reaction of the body as a consequence of another disease. In addition, incorrect ventilation settings can lead to lung injury that corresponds to the clinical picture of ARDS [1]. Monitoring the gas exchange spatially might enable early detection and prevention of complications in mechanical ventilation. Mainly the ratio of ventilation and perfusion contains the necessary information which is needed for determination on how well the gas exchange of the patients work. A simultaneous monitoring of ventilation and perfusion would also allow to optimize or even potentially automize ventilator settings. This improves the patient's chances of survival and prevents long-term damage. Research in the field of Electrical Impedance Tomography (EIT) has shown that EIT has great potential to be used as a monitoring system in ventilated patients. In addition, it has been shown that it is possible to adjust ventilator settings through the guiding of EIT [3].

Promising results for the representation of perfusion were achieved in studies in which an indicator was used to determine the relative pulmonary blood flow (rBF). The reconstructed images showed strong similarity to PET Scans [4]. However, ventro-dorsal gradients in EIT reconstructions did systematically underestimate dorsal regions compared to PET. Though, the gradient information might be important for an accurate diagnosis. In previous studies, the comparison of different approaches of calculating regional blood flow parameters was missing. In this paper we will investigate whether a regularized deconvolution approach using Tikhonov regularization improves the determination of rBF by EIT for clinical application. A regularized reconstruction of pulmonary hemodynamic transfer functions can be better adapted to the inverse problem and might be more robust to noise compared to the maximum slope method and therefore depends less on signal preprocessing. 


\section{Methods}

\subsection{Data and Maximum Slope method}

The data from the preclinical study (approved by the State of Saxony, Germany: (DD24-5131/354/64)) presented in [5] was used for this work. For the evaluation in the presented work a $10 \mathrm{ml} \mathrm{NaCl}$ indicator with a concentration of $10 \%$ was injected intraveneously during mean respiratory hold phases of two experimental states (unilateral ventilation of the right lung (ULV) and bi-lateral ventilation during an optimal PEEP (BLV)). The temporal voltage signal of each measurement $\mathrm{i}=[1, \ldots, 208]$ is filtered with a low-pass butterworth filter $\left(f_{\text {cuto } f}=0.8 \mathrm{~Hz}\right)$ to remove the cardio-synchronous pulsatile component. Additionally, a slow drift signal is removed as explained in [6]. Afterwards a normalized time difference EIT reconstruction with a one-step Gauss-Newton reconstruction was performed [7]. The reconstruction resulted in $\mathrm{k}=[1, \ldots, 1024]$ temporal indicator signals $b_{k}(t)$ representing the temporal impedance signal of each pixel. The signal $b_{k}(t)$ in each pixel $\mathrm{k}$ was used to compute the blood flow parameter $\mathrm{rBF}$.

The newly developed method using regularized deconvolution with Tikhonov regularization is compared with the maximum slope method. The maximum slope method is presented in detail in [4].

\subsection{Regularized Deconvolution}

The idea behind the determination of $\mathrm{rBF}$ by a regularized deconvolution with a Tikhonov regularization is that the lung can be modeled as a system with an arterial blood flow input (AIF) and multiple outputs at different spatial positions within the lung. The transfer function $r_{k}(t)$ between the signal at a spatial pulmonary position $b_{k}(t)$ and the AIF $a(t)$ contains the information about the hemodynamic transfer behavior of this system. The pulmonary blood flow rBF corresponds to the maximum of the transfer function [8]. Fig. 1 shows the AIF and two indicator signals $b_{k}(t)$. It can be seen that the indicator signals correspond to shifted and dispersed versions of the AIF. Consequently, transfer functions are expected to have Gaussian bell like shapes. The goal is to calculate the transfer function from the known AIF $a(t)$ and the measured bolus signal $b_{k}(t)$ in the individual pixels.

The indicator signal $b_{k}(t)$ in each pixel results from the convolution of the input signal $a(t)$ with the transfer function $r B F \cdot r_{k}(t)$ of the system [8]:

$$
b_{k}(t)=r B F \cdot \int_{0}^{\infty} a(\tau) \cdot r_{k}(t-\tau) d \tau=a(t) * r_{k}(t)
$$

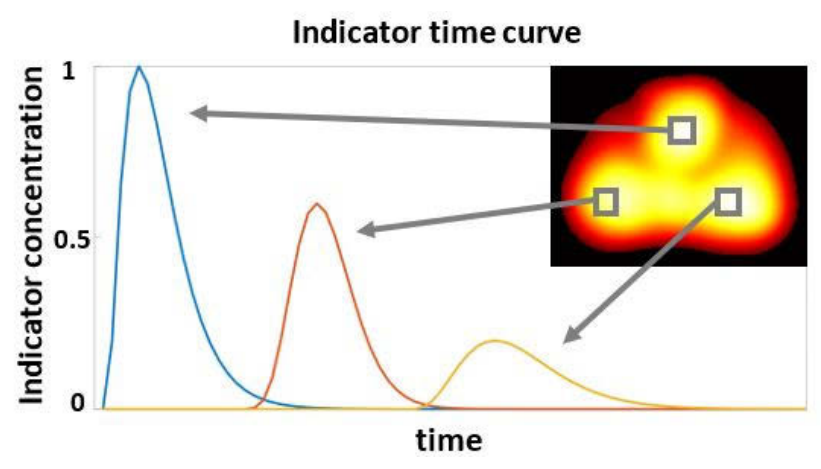

Fig. 1: The blue curve shows the AIF and indicator signals of different pixels in the lung region are displayed by the orange and yellow curve.

It follows that the transfer function $r_{k}(t)$ can be computed using the inverse operator of the convolution. An AIF was estimated in this work from the known impedance curves. For this purpose, the signals of the heart region were selected by image processing and the signal with the largest maximum was selected as AIF. In order to apply the Tikhonov regularization, the AIF was reformulated to a matrix multiplication $A \cdot r=b$ [8]:

$$
\left(\begin{array}{cccc}
a\left(t_{1}\right) & 0 & \ldots & 0 \\
a\left(t_{2}\right) & a\left(t_{1}\right) & \ldots & 0 \\
\ldots & \ldots & \ldots & \ldots \\
a\left(t_{N}\right) & a\left(t_{N-1}\right) & \ldots & a\left(t_{1}\right)
\end{array}\right) \cdot\left(\begin{array}{c}
r_{k}\left(t_{1}\right) \\
r_{k}\left(t_{2}\right) \\
\vdots \\
r_{k}\left(t_{N}\right)
\end{array}\right)=\left(\begin{array}{c}
b_{k}\left(t_{1}\right) \\
b_{k}\left(t_{2}\right) \\
\vdots \\
b_{k}\left(t_{N}\right)
\end{array}\right)
$$

Thus, the linear system of equations needed to determine the transfer function was established. In total, $\mathrm{k}=1024$ equations resulted for a complete image, since each pixel $\mathrm{k}$ has its own transfer function, whereby the AIF remains the same for all pixels.

Prior to the transfer function calculation, the measured indicator signals are shifted by $T_{\text {extra }}=7 \mathrm{sec}$ in time. Since the AIF and indicator signals do overlap extensively, the time shift of the indicator signals is necessary to prevent underestimating of the $\mathrm{rBF}$ due to the deconvolution operation. By increasing the time difference of the signals, the maximum of the transfer function is also shifted and a mapping of the complete transfer function left of the maximum becomes possible.

The Tikhonov regularization for determining the transfer function is [9]:

$$
r_{\lambda}=\operatorname{argmin}\left\{\|A \cdot r-b\|_{2}^{2}+\lambda^{2}\|L \cdot r\|_{2}^{2}\right\}
$$

With regularization parameter $\lambda$ and regularization operator $\mathrm{L}$. Equation (3) is solved for $r$ as follows [9]:

$$
r_{\lambda}=\left(A^{\prime} \cdot A+\lambda \cdot L\right)^{-1}+A^{\prime} \cdot b
$$

To solve equation (4) for $r$, the parameter $\lambda$, which controls the weight of imposed regularization, was automatically 

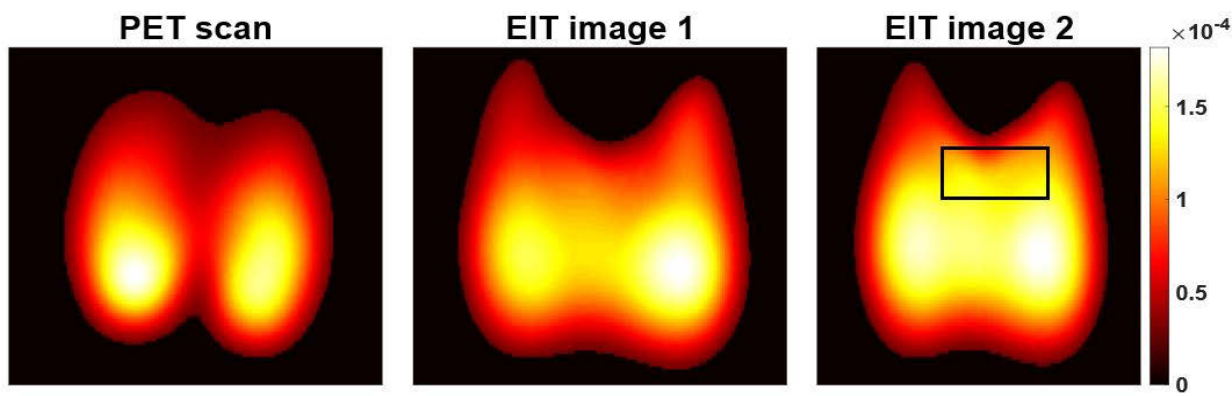

Fig. 2: The images show the parameter rBF for state BLV. For EIT image 1 regularized deconvolution was used to determinate $\mathrm{rBF}$. EIT image 2 shows the rBF determinated by the maximum slope method. optimized. The function fminsearch provided by MATLAB R2019a was used to minimize the error norm $(\rho)$ and the solution norm $(\eta)$ of $\mathrm{r}$. The calculation of $\rho$ and $\eta$ is described in [9]. The Pseudocode representing the algorithm used for optimizing $\lambda$ is displayed in algorithm 1 .

Although the criterion depends on the scaling, the method performed more robust than an optimization by determining the maximum curvature (L-curve criterion) [9].

\section{Algorithm 1 Optimizing $\lambda$}

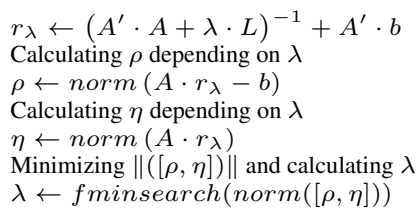

In a first step, all spatial $r_{k}(t)$ were reconstructed using the presented method for $\lambda$ optimization and the pseudoinverse of equation (4) with the identity matrix as regularization operator $\mathrm{L}=\mathrm{I}$. Then, the correlation between the resulting transfer functions $r_{k}(t)$ and simulated transfer functions $g_{k}(t)$ was determined. For each pixel the transfer function was simulated through a gaussian distribution. The transfer functions were calculated as follows:

$$
g_{k}=\left(\max (a(t))-\max \left(b_{k}(t)\right)\right) \cdot e^{-\frac{1}{2} \cdot\left(\frac{t-T_{\text {extra }}}{T_{\text {extra }}}\right)^{2}}
$$

From the correlation coefficients for each individual pixel a diagonal matrix $D_{\text {corr }} \in \mathbb{R}^{1024 \times 1024}$ was constructed. The regularization operator $L_{\text {corr }}$ was derived from the correlation matrix and the transfer functions to $L_{\text {corr }}=D_{\text {corr }} \cdot r^{T}$.

In order to ensure numerical stability, a projection on an orthogonal subspace was performed. The Tikhonov regularization was performed with the pseudoinverse of the operator $L_{\text {corr }}$. The pseudoinverse $L_{A}^{+}$of $L_{c o r r}$. is calculated as follows [10]:

$$
L_{A}^{+}=\left(I-\left(A\left(I-L_{\text {corr }}^{+} L_{c o r r}\right)\right)^{+} A\right) L_{c o r r}^{+}
$$

$L_{\text {corr }}^{+}$represents the Moore-Penrose inverse of $L_{c o r r}$.
The new formulation of the Tikhonov regularization is [10]:

$$
\bar{r}_{\lambda}=\operatorname{argmin}\left\{\left\|A \cdot L_{A}^{+} \cdot \bar{r}-b\right\|_{2}^{2}+\lambda\|\bar{r}\|_{2}^{2}\right\}
$$

The transfer function was calculated using the solutions of equation (6) and (7):

$$
r_{\lambda}=L_{A}^{+} \cdot \bar{r}_{\lambda}
$$

\subsection{Comparison to PET}

Finally, the EIT images were compared with the PET scans. Prior to the comparison, EIT images and PET scans were normalized to yield one as the sum of all rBF values of one image. For the comparison, the lung regions of the images as obtained by CT were divided into eight spatial regions and the correlation was determined for these spatial values. A detailed description can be found in [6].

\section{Results}

Table 1 shows the image correlation between EIT and PET scan. The correlation is very similar for both approaches. For $\mathrm{BLV}$, a median correlation of 0.912 is achieved with the maximum slope method, and a median of 0.935 is achieved with the regularized deconvolution using Tikhonov regularization. For state ULV the correlation of the EIT images with the PET scan differ slightly, the median for the maximum slope method is 0.862 , with the regularized deconvolution the median of correlation is 0.863 .

Tab. 1: Correlation EIT und PET.

\begin{tabular}{lcccc}
\hline \hline & \multicolumn{2}{c}{ ULV } & \multicolumn{2}{c}{ BLV } \\
\cline { 2 - 4 } & Median & IQR & Median & IQR \\
\hline $\begin{array}{l}\text { Maximum } \\
\begin{array}{l}\text { Slope } \\
\text { Regularized } \\
\text { deconvolution }\end{array}\end{array}$ & 0.862 & 0.148 & 0.912 & 0.113 \\
\hline \hline
\end{tabular}


Fig. 2 shows the reconstructed EIT images and the PET scan for the BLV state. In contrast to the quantitative analysis, there are clear differences in the images. The EIT image reconstructed with the regularized deconvolution shows a better separation of the lung regions compared to the image reconstructed with the maximum slope method. The framed region of the EIT image reconstructed with maximum slope method shows leftovers of the heart regions which could not be eliminated by the heart region filter. This is an indication that the region separation actually works worse with this approach and is not caused by a bad colormap. At the same time a difference can be seen in both images compared to the PET scan when comparing the strength of perfusion in the right and left lung.

In addition to the image reconstruction, the computing time for the different reconstruction methods was compared. For the regularized deconvolution method, the average calculation time for the examined animals was 163.4 seconds, in comparison the computing time for the maximum slope method was on average 2.11 seconds.

\section{Discussion}

The quantitative comparison of EIT images and PET scans in the previous section showed that the methods investigated led to a good correlation of EIT images and PET scans. On the other hand, the investigation of the runtime showed that the computing time for regularized deconvolution with Tikhonov regularization is about the factor 77 larger than with the maximum slope method.

A clear improvement of the spatial similarity to PET using the regularized deconvolution reconstruction could not be observed. Furthermore, regularized deconvolution using Tikhonov regularization implies a substantially higher computation time. However, it should also be taken into account when evaluating the results that image reconstruction depends on many more factors than the methods examined here. A decisive factor influencing the results is the estimation of the AIF, which in turn depends on the determination of the heart region. An improvement of the AIF or an improved determination of the cardiac region could thus also lead to an improvement of the estimated transfer functions.

In addition, consideration should be given to changing the method of comparison of PET scans and EIT images. In the images were significant differences between the different reconstruction methods. In contrast, there are hardly any measurable differences in the correlation values. It is therefore quite possible that a quantitative comparison using other parameters, such as the ventro-dorsal gradient, can lead to measurable differences. To make the Tikhonov regularization more interesting for the application, it would also be interesting to investigate whether it is possible to determine a $\lambda$ on the basis of existing data, which can be used for all further upcoming reconstructions of different data. This would eliminate the timeconsuming optimization of lambda and reduce the computing time considerably.

\section{Author Statement}

Research funding: The Institute of Biomedical Engineering received funds from Drägerwerk AG \& Co. KGaA for the cooperation project of Michael Kircher. The animal study was funded by Drägerwerk AG \& Co. KGaA.

\section{References}

[1] Keddissi, J I, McCaffree, D R Acute Respiratory Distress Syndrome. In: Jindal S, editor. Handbook of Pulmonary und Critical Care Medicine. New Dehli: Jaypee Brothers Medical Publishers; 2019:507-513.

[2] Akutes Atemnotsyndrom, Infographik (Zugriff: 17.11.2019). https://www.europeanlung.org/de/lungenkrankheitenand-informationen/lungenkrankheiten/akutesatemnotsyndrom(ards).

[3] Frerichs I, Becher T, Weiler N. Electrical impedance tomography imaging of the cardiopulmonary system. Current Opinion in Critical Care 2014;20:323-232.

[4] Borges J B, Suarez-Sipmann F, Bohm S, Tusman G, Melo $A$, Maripuu $E$, et al. Regional lung perfusion estimated by electrical impedance tomography in a piglet model of lung collapse. Journal of Applied Physiology 2012;112:225-236.

[5] Bluth T, Kiss T, Kircher M, Braune A, Bozsak C, Huhle R, et al. Measurement of relative lung perfusion with electrical impedance and positron emission tomography: an experimental comparative study in pigs. British Journal of Anaesthesia 2019;123:246-254.

[6] Kircher M, Braune A, Bluth T, Gama de Abreu M, Dössel O, Stender B. Method for comparing pulmonary perfusion measured by PET/CT and indicator based EIT measurements in a porcine model. 19th International Conference on Biomedical Applications of Electrical Impedance Tomography 2018.

[7] Teschner E, Imhoff M, Leonhardt S. Electrical Impedance Tomography: The realization of regional ventilation monitoring. 2nd ed. Lübeck: Drägerwerk AG und Co. KGaA; 2015.

[8] Ostergaard L, Weisskoff R, Chesler A, Gyldensted C, Rosen B. High Resolution Measurement of Cerebral Blood Flow using Intravascular Tracer Bolus Passages. Part I: Mathematical Approach and Statistical Analysis. Magnetic Resonance in Medicine 1996;36:715-725.

[9] Hansen P C. The L-curve and its use in the numerical treatment of inverse problems. In: Johnston P, editor. Computational inverse problems in electrocardiography. Boston: WIT Press; 2001:119-142.

[10] Morigi S, Reichel L, Sgallari F. Orthogonal projection regularization operators. Numerical Algorithms 2007;44:99-114. 